\title{
Risk Perception, Media Exposure, and Visitors' Behavior Responses to Florida Red Tide
}

\author{
Ignatius Cahyanto \& Bingjie Liu-Lastres
}

2020

\begin{abstract}
Florida's Red Tide outbreak, a major environmental disturbance in 2018, not only garnered nationwide attention but also affected both in-state and out-of-state visitors. Guided by the Social Amplification of Risk Framework (SARF), this study examined the relationships between media exposure, risk perception, and visitors' behavioral responses. Data were gathered from two surveys in late 2018. The findings validate the practicality of applying SARF to the current context. This study also found that both perceived consequences and access to the community are significant predictors of visitor behavior. This study further discussed how to market destinations during turbulent times.
\end{abstract}

Keyword: Red Tide, Florida, Tourism, Risk, Media, SARF, HAB, Destination Marketing Word Count: 7281

This is the author's manuscript of the work published in final edited form as:

Cahyanto, I., \& Liu-Lastres, B. (2020). Risk perception, media exposure, and visitor's behavior responses to Florida Red Tide. Journal of Travel \& Tourism Marketing, 37(4), 447-459. https://doi.org/10.1080/10548408.2020.1783426 


\section{Introduction}

Environmental disturbances, including hurricanes, earthquakes, and wildfires, recur regularly and can significantly impact destinations (Cahyanto et al., 2016). The tourism system is vulnerable as these disturbances can disrupt the ecological system of a destination, devastate planned vacations, engender tourists' disappointment, and foster negative impressions of a destination (Thapa et al., 2013). Thus, destinations bear multiple responsibilities, including managing and marketing destinations at all times and ensuring the health and safety of residents and visitors (Wilks, Stephan, \& Moore, 2013).

Many factors may affect visitors' risk perception and their subsequent protective behaviors (e.g., traveling to a safer destination, canceling the trip). At the same time, scholars pointed out that the geographical distance from the incident plays a critical role, with the further the individuals are from the event, the more likely they are to modify their travel plans (Cahyanto \& Pennington-Gray, 2015; Kozak, Crotts, \& Law, 2007). Those with stronger ties to and geographical knowledge of a destination, however, are more resistant to negative images of that destination (Cahyanto \& Pennington-Gray, 2015).

Communication with the target market is vital to destination marketing. Still, during environmental incidents it can be challenging because media coverage typically focuses on negative aspects of the events, which can amplify risk perception and cause visitors to avoid the destination (Liu \& Pennington-Gray, 2015; Ratzan \& Moritsugo, 2014). Thus, understanding 
how various levels of media exposure influence individual risk perception and protective behaviors becomes crucial, as it will aid in developing a critical targeted media crisis strategy. Yet, there is a need to further understand how exposure to media reports of crisis affects travel plans to the destination being reported on, especially in the era of ubiquitous media (Liu \& Pennington-Gray, 2015).

A significant environmental crisis that impacted the State of Florida in 2018 was the Red Tide outbreak in the Gulf of Mexico. Red Tide is highly concentrated blooms of microscopic algae, Karenia brevis, which occurs annually along Florida's coastline and sporadically in other Gulf Coast states. Despite the extensive literature on Red Tide, there is a dearth of studies on public perception of Red Tide events (Kuhar et al., 2009). Understanding the public's perceptions on the issue, particularly visitors to Florida, given its popularity as a tourism destination, is crucial, since perception drives how individuals respond and behave relative to risk events (Cahyanto et al., 2016). Nonetheless, behaviors may not be commensurate with the actual risk surrounding the event. Individual behaviors, such as avoiding a destination, can influence the economic impacts of Red Tide. These behaviors are passed on through social networks, resulting in significant economic losses to tourism industries, as negative media coverage is a major cause of adverse consumer reactions such as canceling a visit to a destination (Hoagland et al., 2014).

Kasperson et al. (2003) described this phenomenon as the "social amplification of risk", whose effects can be further explained by the Social Amplification of Risk Framework (SARF). The framework asserts that the social and economic impacts of a risk event are not merely the result of the direct impacts of the event. What is often more crucial is the interface of psychological, cultural, social, and institutional practices that amplify or attenuate how the public 
experiences a risk, which results in secondary impacts (Kasperson et al., 2003). In a later development, Masuda and Garvin (2006) emphasized the role of place and culture in the perception of risk by arguing that place is central in understanding individual risk perception.

This study incorporated the SARF and the role of place-specific context (Kasperson et al., 2003; Rickard et al., 2013) in order to explore visitors' perceptions of and behavioral responses toward Red Tide. Transient populations' (e.g., visitors) perceptions and their subsequent reactions to Red Tide have not been thoroughly investigated (Nierenberg et al., 2010). Consequently, the primary objective of this study is to explore the relationships between the perceptions of the risk, the role of media exposure to Red Tide, and the likelihood to engage in protective behaviors. To that end, this article is framed by the following interrelated questions:

1. What is the relationship between visitors' place of residence and risk perceptions of Florida Red Tide?

2. What is the relationship between media exposure and visitors' risk perceptions of Florida Red Tide?

3. What are the major influencers of visitors' behavioral changes in response to Florida Red Tide?

\section{Situational Context}

The Red Tide outbreak in the Gulf of Mexico coastal areas was a major environmental disturbance with widespread consequences. Red Tide is part of Harmful Algal Blooms (HAB) that can generate neurotoxins that threaten human and marine organisms through ingestion and inhalation of the toxins (Steidinger, 2009). Humans are at risk from neurotoxic shellfish poisoning (NSP) or paralytic shellfish poisoning (PSP), both of which are caused by consuming 
affected shellfish (Hoagland et al., 2014). Red Tide cells release toxins into the air and cause airborne illnesses resulting in respiratory problems, nausea, diarrhea, lack of motor coordination, pupil dilation, tingling of fingers and toes, and the reversal of hot and cold sensations (Diaz et al., 2019).

The 2018 Red Tide has endured since November 2017, making it the worst Red Tide since 2006. By October 2018, hundreds of manatees, dozens of dolphins, thousands of fish, and over 300 sea turtles died or washed along shores in putrid-smelling masses (Resnick, 2018). Resultant beach closures threatened tourism in Florida, which is a top U.S. destination that welcomed 116.5 million visitors in 2017 (Visit Florida, 2018). 94 percent of Southwest Florida businesses suffered Red Tide-related economic losses (Florida Division of Emergency Management, 2018), and, in August of 2018, Florida's Governor declared a state of emergency, allocating millions of dollars for cleanup and response (Sims, 2018).

Media coverage of Red Tide has centered on risk, including environmental, tourism, and public health risks (Li et al., 2015). A direct relationship between increased media exposure and decreased touristic activities occurred during Red Tide events (Morgan, Larkin, \& Adams, 2011). To mitigate negative repercussions and sustain local tourism, there is a need to understand visitors' behavioral responses toward such media coverage while also promoting self-protective behavior (Nierenberg et al., 2010). Despite this need, most Red Tide studies have focused on the biological characteristics of HABs (Kuhar et al., 2009; Nierenberg et al., 2010) and tourism has not been a focus. How risk perception influences individuals' health-related behavioral changes (Brewer et al., 2007) and travel-related behavioral changes (Liu-Lastres, Schroeder, \& Pennington-Gray 2018) is underexplored to date. Consequently, the 2018 Florida Red Tide 
provides an ideal context to explore the relationship between media exposure, risk perception, and visitors' behavioral responses.

\section{Literature Review}

\section{Social Risk Amplification Framework (SARF)}

SARF was developed in the 1980s to explain how risk is perceived, shaped, and communicated in contemporary society (Kasperson et al., 1988). SARF holds that there are often glaring distinctions between technical risk assessment and assessment of risk by individuals. Hazards deemed as low risk by experts can nonetheless generate great social attention or risk amplification, while high-risk hazards often generate much less social attention (Kasperson et al., 2003). SARF deciphers how diverse hazardous events interact with psychological, social, institutional, and cultural processes, leading to intensification or attenuation of risk perceptions and related behaviors (Pidgeon, Kasperson, \& Slovic, 2003).

According to SARF, amplification or attenuation begins either with an event or awareness of a hazard. Signals generated during events blend facts, values, and symbolic meanings, which are intensified during a transmission process as signals pass through various individual and social stations (Kasperson et al., 1988; Kasperson, 2012). Ripple effects may also occur where the influence of the event magnifies far beyond the initial risk and moves beyond temporal, sectoral, or geographical restrictions (Kasperson et al., 2003). These ripple effects can impact local communities, stakeholders, and society at large and include financial losses, regulatory actions, organizational changes, litigation, increased physical risks, community concerns, and the erosion of public confidence (Kasperson \& Kasperson, 2001). 
SARF deems the news media as a key social station of risk amplification and attenuation that influences public perception of risk and behavioral responses (Kasperson \& Kasperson, 1996). The news media engage in several key amplification steps: filtering and decoding risk signals, processing risk information, and attaching social values to information (Kasperson et al., 1988). These steps lead to the transformation of risk signals, which can increase or decrease the salience of certain aspects of risk messages (Pidgeon et al., 2003). Conventional signal transformation processes include giving disproportionate, long term attention to some risks while disregarding others, legitimating some points of perspectives more or less than others, and exaggerating the nature of some risks but not others. These media responses shape public discourse, and large volumes of information and repeated stories serve to mobilize latent fear and amplify risk (Kasperson et al., 1988). Equally important are the symbols, metaphors, and discourses used to portray and characterize risk in the news media (Pidgeon et al., 2003). The news media can amplify risks and shorten or prolong the lasting impacts of a crisis (Rubaltelli et al., 2018; Chong \& Choy, 2018).

Although SARF has been lauded for explaining how risk can be amplified through mass media, it has been criticized as ambiguous. The framework has been faulted for not being a useful instrument for predicting variations over time, mainly because SARF does not provide any testable hypotheses to demonstrate the social amplification process (Wirz et al., 2018). Similarly, Duckett and Busby (2013) argued that there is an absence of a clear-cut criterion for discriminating between amplification and attenuation.

Tourism research grounded in SARF has been limited in scope and is sparse, primarily because it is difficult to empirically test the overall guidelines (Wirz et al. 2018; Raude et al. 2004). One exception is a study conducted by Shakeela and Becken (2015), which examined 
tourism leaders' perceptions of risks of climate change and their policy-making processes in the Maldives. The study found SARF useful to understand the rationale behind decision-making; however, it did not identify the key elements that affect stakeholders' decision-making. Despite such findings demonstrating its usefulness, SARF has not been empirically validated in the tourism management context, largely because it is difficult to empirically test the overall guidelines (Wirz et al. 2018; Raude et al. 2004). In the same vein, the application of SARF in understanding the interplay among visitors' media exposure, risk perception, and behavioral responses in the context of environmental disturbances remains underexplored.

\section{Risk Perception and Visitors' Behavioral Responses}

In tourism research, risk perception has received significant attention in recent years (Karl, 2018). Travel risk research studies increased after a series of major crises in the early 2000 s, including the 9/11 terrorist attack in 2001, the 2003 SARS outbreak, and the 2004 Indian Ocean earthquake and tsunami. Media coverage of these events resulted in an increased public focus on risks (Liu \& Pennington-Gray, 2015). Researchers recognize the impact of risk perception on travelers' behavior, and as travel risk research has progressed, it has become evident that risk can be further divided into various types, ranging from issue and destination to travel-specific risks. The increased emphasis on travel risk perception and its impacts is highly consistent with the SARF framework.

Another interest to tourism scholars and practitioners involves visitors' risk reduction strategies (Jonas \& Mansfeld, 2017), which is salient for destinations experiencing emerging public health issues, as visitors are often vulnerable to threats and can accelerate the spread of a potential health epidemic (Richter, 2003). Promoting visitors' self-protective behavior is an 
important initiative to address such issues (Wang et al., 2019). Information-gathering is considered crucial in reducing visitors' perceived risk and increasing their likelihood to employ self-protective behaviors (Jonas \& Mansfeld, 2017). This aligns well with SARF, which emphasizes the importance of media coverage on individuals' perceptions and behavioral responses to risk events (Kasperson et al., 2003). The media is a major player in risk perception, and as such, Renn (1991) referred to media outlets as being social stations that amplify risk. Burns, as cited by Wirz et al. (2018), argued that the media's behavior and its interplay with the public is a defining factor in what the impact of a hazardous event will be.

Notably, studies examining people's risk perceptions regarding Red Tide and HAB found that place of residence affected risk perception of Red Tide, wherein Floridians tend to perceive the issue as more severe than out-of-state visitors (Kuhar et al., 2009; Nierenberg et al., 2010). Kuhar et al. (2009) attributed Floridians' higher risk perception to greater news exposure within the state. Likewise, Li et al. (2015) confirmed that Red Tide is a common topic in Florida's local news, which highlights the risks. Thus, there is a need to explore the complexity of perceptions of Red Tide to assist in the development of appropriate control and mitigation strategies.

\section{Methodology}

\section{Data Collection}

Prior to the data collection, a preliminary study was conducted in mid-2018 to obtain insights into (1) visitors' risk perceptions of the 2018 Florida Red Tide and (2) visitors' behavioral changes in response to Red Tide. Based on the literature and the results of the preliminary study, a questionnaire was created and tested. The data were collected through two waves of online panel surveys in late 2018. The first survey consists of a U.S. sample of potential out-of-state 
visitors (18 years and older) who planned to visit Florida in the following six months. A total of 969 completed surveys were used for this study. The second wave of the survey utilized Florida residents who planned to visit other parts of Florida (more than 50 miles from their residency) for leisure purposes and was comprised of 456 completed surveys. The two-wave designs were used to include specific questions relevant to each market while allowing for comparison in aspects that were relevant to both markets. Both samples were compared with the typical demographics of Florida visitors provided by Visit Florida, the marketing arm of Florida (Visit Florida, 2018). There were no significant differences between the samples regarding the key population demographics, indicating the suitability of the samples.

\section{Data Measurement}

Both surveys included questions related to general perceptions of Red Tide, risk perception, media use, media exposure, risk probability, risk consequences, news attention, access to community, demographics, and other travel behavior items. In this study, four key aspects were defined. First, perceived risks, concerns, and subjective knowledge of Red Tide were measured by statements with a 5-point Likert scale (strongly disagree to strongly agree). Items were adapted from Kuhar et al. (2009) and Nierenberg et al. (2010). To measure subjective knowledge, respondents were asked to indicate their confidence in explaining Red Tide and its impacts to their friends and relatives on a 10-point scale and were asked to identify areas impacted by Red Tide on a map.

The second aspect of the survey measured media exposure by asking participants to rate their level of exposure to Red Tide news from six media outlets on a 5-point Likert scale (never to always). The items were adapted from Lin and Lagoe (2013). Respondents were asked about 
the level of attention they gave to news about Red Tide in Florida on a 5-point Likert scale (none to a great deal). The third aspect of the survey measured respondents' access to the local community. This construct gauges the extent of reliance on information from family and friends, tourism organizations, local residents, and local governments in the destination should an outbreak occur during their trip using a five-point Likert scale. The fourth aspect measured the likelihood of engaging in behavioral modifications due to Red Tide, by measuring two behaviors: (1) changing an itinerary to exclude a visit to the beach; (2) changing an itinerary to visit alternative destinations in Florida.

\section{Data Analysis}

Data analysis was conducted in multiple phases. First, a series of descriptive analyses were conducted to check the normality of variables in both surveys and to examine respondent profiles. Upon confirming the normality of the variables, both surveys were merged into a single dataset to allow for multiple comparisons. Next, respondents were grouped based on media exposure associated with Red Tide news. Hierarchical cluster analysis was used to identify the number of clusters. Subsequently, K-means cluster analysis was used to classify the samples based on the respondent's sensitivity to media exposure related to Red Tide. The K-means clustering method produces results that are less receptive to outliers in the data, the distance measure used, and the inclusion of irrelevant variables (Hair, 2011). Then a discriminant analysis was employed to confirm the number of clusters. Segment characteristics were delineated by various univariate and multivariate statistical procedures. Exclusively on the identified clusters, the difference of perceptions and perceived risks associated with Red Tide was assessed by 
ANOVA and Chi-square analysis. Cluster analysis was used for validating and profiling (Park \& Yoon, 2009).

Lastly, to identify the major influencers on the samples' behavioral changes, six separate ordered response models were employed to relate the likelihood of engaging in protective behaviors to the independent variables. The ordered response model recognizes the inherent ordering in the outcome variables of interest and allows for the calculation of the probability of each level of an outcome as a function of explanatory factors (McKelvey \& Zavoina, 1975). All statistical analyses were performed using the SPSS 23 package.

\section{Results}

\section{Respondents Profiles}

Regarding the out-of-state visitors, out of 969 individuals, $49.5 \%$ were $25-34$ years old; $53.5 \%$ were male; $68 \%$ had a bachelor's degree; $57.9 \%$ were married; $49.3 \%$ had no children, and $53 \%$ had heard of Florida Red Tide before. For in-state visitors, 37.5\% were $25-34$ years old; 33.8\% had a bachelor's degree; $40.8 \%$ were married, and more than 50\% did not have children. Table 1 presents a summary of the respondent profiles.

\section{[Insert Table 1 here]}

\section{Residency and Perceptions of Red Tide}

To explore the influence of residency on respondents' perceptions of Florida Red Tide, a series of T-tests were conducted to compare the in-state and out-of-state visitors' perceptions. As shown in Table 2, both groups surmised that Florida Red Tide occurrences are lasting longer and are more severe $(\mathrm{t}=.17, \mathrm{p}=.861)$. Out-of-state visitors believed that Red Tide outbreaks are 
occurring more frequently than in-state visitors $(\mathrm{t}=2.43, \mathrm{p}=.015)$, and are directly affected by urban growth $(\mathrm{t}=4.32, \mathrm{p}=.001)$. Concern about Red Tide was higher among in-state visitors $(\mathrm{t}=6.87, \mathrm{p}=.001)$. Regarding knowledge, in-state visitors showed more confidence in explaining Red Tide to friends and relatives $(\mathrm{t}=7.67, \mathrm{p}=.001)$ and were more accurate in identifying areas severely impacted by Red Tide on the map in Figure 1. In-state visitors also demonstrated a higher perception of the probability of risks regarding consuming contaminated shellfish $(\mathrm{t}=4.13$, $\mathrm{p}=.001)$, respiratory problems $(\mathrm{t}=7.69, \mathrm{p}=.001)$, and the consequences of the risks related to their travel experiences and overall health respectively.

The differences between in-state visitors and out-of-state visitors suggested that in-state visitors amplified their concerns because they are more frequently affected by red tides due to their proximity to the affected areas. In addition, red tides were covered more frequently by the news media in Florida, particularly in coastal areas where impacts are most austere. Furthermore, the results confirmed the pertinence of the role of place-specific factors in understanding risk perception.

\author{
[Insert Table 2 here] \\ [Insert Figure 1 here]
}

\title{
Media Exposure and Perceptions
}

To measure the effects of media exposure, this study segmented the respondents based on media exposure levels and identified three clusters. The results of an ANOVA test indicated that all six media exposure items contributed to differentiating the three-cluster solution $(p<.05)$, and the results of Scheffe tests confirmed the statistically significant differences between clusters (Table 
3). Standardized canonical discriminant function coefficients indicated that the item "online news" was the strongest predictor for Function 1 (.408), while the item "Radio news" was the strongest predictor for Function 2 (.701). Almost all (98\%) of the 1406 grouped cases were correctly classified, signifying a very high accuracy rate. Specifically, low exposure (99.1\%), medium exposure (97.5\%), and high exposure (97.2\%) were correctly classified into their respective groups. Clusters were labeled as (1) Low Media Exposure, (2) Medium Media Exposure, and (3) High Media Exposure. The Low Media Exposure group paid little or no attention to Red Tides news compared to the other two groups. Regarding socio-demographics, there was no significant difference in gender and marital status. Differences were found on residency, age, and education. $71.9 \%$ of the group were out-of-state visitors, with in-statevisitors comprising $28.1 \%$ of the sample. $35-49$ years old comprised $43.2 \%$, and $40.8 \%$ had a bachelor's degree. The Medium Media Exposure group paid casual attention to Red Tides news. $60.7 \%$ of the Medium Media Exposure group were out-of-state-, while in-state visitors comprised $39.3 \%$ of the sample. Those $35-49$ years old comprised $38.7 \%$, and $40.5 \%$ of them had a bachelor's degree. The High Media Exposure group was comprised of those who were highly exposed to Red Tides news and paid attention to such news. $71 \%$ of the members of this group were out-of-state visitors; $29 \%$ were $50-74$ years old, and 52\% had a bachelor's degree.

\section{[Insert Table 3 here]}

To further explore the influence of media exposure on the sample's perceptions, each cluster was cross-examined with several external perception-related variables. As shown in Table 4, the high-exposure group is more likely to believe that Red Tide events are lasting longer and 
are more severe $(\mathrm{F}=10.32, \mathrm{p}=.001)$, that Red Tide is directly affected by urban growth $(\mathrm{F}=3.07$, $\mathrm{p}=.047$ ), and that there should be stricter regulations to prevent coastal pollution and runoff $(\mathrm{F}=9.39, \mathrm{p}=.001)$. The high-exposure group also expressed more concerns over Red Tide $(\mathrm{F}=$ $68.67, \mathrm{p}=.01$ ), and deemed the consequences to be more severe, both on their expected travel experience and personal health. The high-exposure group scored higher on the probability of all risks. Media exposure affected respondents' behavioral responses with the high-exposure group being more likely to exclude a beach visit $(\mathrm{F}=5.25, \mathrm{p}=.006)$ and more likely to visit alternative destinations in Florida $(\mathrm{F}=6.19, \mathrm{p}=.002)$.

\section{[Insert Table 4 here]}

\section{Behavioral Changes}

This study featured two types of behavioral changes: (1) excluding beach visits; and (2) visiting other destinations in Florida, which were the most common behavioral responses reported in the preliminary study. To identify the influencers, the items were subjected to reliability analysis. The reliabilities of four constructs (risk consequence-experience, risk consequence-health, risk probability, and access to the community) were above the recommended value of .70, indicating acceptable internal consistency. A factor analysis was employed to ensure the unidimensionality of each construct.

In the Ordered Response Model, a positive parameter indicated that the corresponding variable was associated with a higher likelihood of performing the behavior. A negative parameter indicated the opposite effect. The parameters of the models were estimated using the maximum likelihood estimator. Split samples based on media exposure levels were created and used to relate several constructs to both dependent variables, resulting in six models. 
As shown in Table 5, the Log-likelihood in all models was significant, indicating a significant improvement from their respective baseline model. Three constructs warranted further discussion in this study: risk consequences of Red Tide on visitors' travel experience, access to the community, and residency of visitors.

For the likelihood of changing one's travel itinerary to exclude a beach visit, risk consequences on travel experience was found to be significant regardless of media exposure levels. Access to the local community was also found to have a significant positive association with both low and high exposure groups. In addition, residency status was found to be significant for both low and high exposure groups, with in-state visitors being less likely to change travel plans to exclude beach visits.

Regarding the likelihood of visiting other Florida destinations, risk consequences on travel experience had a positive association for both low and high exposure groups. Access to the community had a positive relationship with the dependent variable regardless of media exposure levels. In addition, in-state visitors were less likely to visit other destinations in Florida.

[Insert Table 5 here]

\section{Discussion}

This study set out to elucidate the relationship between risk perception, media exposure, and the likelihood of engaging in protective behaviors in the context of the 2018 Florida Red Tide. From a theoretical perspective, this study seeks to validate the application of SARF in the context of environmental disturbances (e.g., Red Tide) and transient populations (i.e., visitors). From a practical perspective, this study aims at helping to inform further projects to encourage 
visitors to engage in protective behaviors and on how to best mitigate the adverse health and societal impacts associated with environmental disturbances on destinations.

The first research question examined the public's perception of Red Tide. Interestingly, the overall perception shared by both in-state and out-of-state visitors is similar, wherein they deem Red Tide to be lasting longer and increasing in severity. The findings also revealed group differences, with in-state visitors possessing more knowledge about Red Tide, but still perceiving Red Tide as threatening to their incoming trips and own health. This is consistent with the findings of previous studies (Kuhar et al. 2009; Neirenberg et al. 2010). The variation in perceptions may be explained by Florida residents' direct experience with Red Tide coupled with the extensive local news coverage. Because the 2018 Red Tide lasted longer than in previous years, there has been greater media coverage and public discussion surrounding the issue, resulting in in-state visitors' growing concerns.

The findings of this study also underscore the critical role played by residency in SARF. Kasperson (2012) pointed out the importance of social influences during the risk amplification/attenuation process. In the same vein, various social elements have been explored in the SARF, such as gender, occupation, and age. Masuda and Garvin (2006) argued that there is a need to assess how spatial dimensions may cause individuals or groups to amplify or attenuate risks. The findings of this study provide further support to this argument, as this study noted that the public's perceptions and concerns of Red Tide vary according to residency. Instate visitors exhibit greater concern regarding Red Tide, despite their extensive knowledge about the issue. Such differences may be explained by the concept of place attachment, which is related to one's family, work, leisure, and other interactions of daily life activities and place (Buttimer, 1993). Due to the special meaning that place holds for individuals, tensions can 
become noticeable regarding emerging local issues (Martin 2003). As such, residency and place attachment are helpful in explaining the variation of perceived risks between in-state and out-ofstate visitors. Meanwhile, place attachment can be deemed as a significant element in both reinforcing and reflecting the social construction of risk, which needs further exploration.

The second study question measured the effects of media exposure on the respondents' perceptions and behavioral reactions. The findings confirm the influence of media exposure, as the high-exposure group demonstrated higher risk perception towards Red Tide and is more likely to adopt behavioral changes. SARF suggests that the assessment of a risk event can be amplified by different stations, ranging from persons to groups and organizations who transform and disseminate information about the hazard; therefore, the risk information and the way it is disseminated can create 'ripples' through society (Kasperson 2012). In the meantime, previous research (Li et al., 2015) found that the local news about Red Tide tends to focus on the negative consequences. Thus, taken all together, it becomes apparent that the high amount of media exposure and the skewed media content serve as a risk amplifier. As such, the audiences under the high-exposure condition not only possess a higher level of perceived risk but also demonstrated a stronger likelihood to avoid the threats and modify their travel plans.

To better understand the drivers of the respondents' behavioral changes under different media exposure conditions, this study tested the effects of different variables on visitors' decisions to (1) exclude beach visits in their visit and (2) visit other destinations in Florida. Firstly, this study found that the perceived consequence of travel experience is a significant predictor of both dependent variables. Previous research on tourists' health risk perception tended to focus on consequences related to personal health, such as illness and symptoms (e.g., Chien et al., 2016; Wang et al., 2019), while the findings of this study showed that the respondents also 
expressed concern about the potential negative impacts on their travel experiences. This is suitable for the current context but has been overlooked in past studies. The concern that Red Tide would affect travel experiences is understandable as leisure travelers tend to invest their time, money, and other resources for their travel to ensure they have high-quality travel experiences. Red Tide would therefore negatively affect their experience should the beach be closed or if they cannot visit the planned destination due to Red Tide. Thus, to ensure they save their 'investment,' it is understandable that they would engage in protective behaviors. Future studies should explore the extent to which such trade-off occurs in the mind of visitors.

Additionally, access to the community is a significant predictor of both dependent variables in the high-exposure condition. Access to the local community can be interpreted as visitors' reliance on local friends/relatives, local residents, local tourism offices, and local governments. Stronger ties to local resources are associated with greater access to information and knowledge, which, in turn, leads to more informed decisions. In other hurricane evacuation studies, it has been noted that individuals do not necessarily base evacuation decisions on the first information they receive from media sources. Instead, people typically confirm the information with their social networks, such as friends and relatives, before acting upon it (Lindell, Lu, \& Prater 2005). Similarly, visitors tend to confirm the information with local sources before making a final decision (Cahyanto and Pennington-Gray, 2015). Thus, ensuring a transparent and local-based information flow among visitors has become essential in this context.

Lastly, residency was found to be a significant predictor of both dependent variables in the high-exposure condition. This corresponds well with our previous observation involving access to the community. Compared to out-of-state visitors, in-state visitors have greater access to local resources and to the local community. Also, as reported earlier, they have more knowledge about 
Red Tide, albeit heightened risk perception. Thus, they are more likely to adopt behavioral changes to be risk aversive.

\section{Practical Implementations}

With the increasing incidence of environmental disturbances such as climate change, Red Tide, and wildfires, many destinations are facing the challenge of managing a destination in unsettling times. Sometimes the situation may worsen due to the news impact and the public's lack of insights and knowledge (Avraham \& Ketter, 2008; Liu \& Pennington-Gray, 2015). The practical implications of this study are to provide insights and tools to destinations to better manage such situations. To start with, destinations should be aware that the high amount of media exposure surrounding emerging issues naturally results in heightened public concern and misperceptions, which is difficult to correct. As in this study, most in-state visitors have more knowledge about Red Tide, yet they still see it as a threat to their incoming trips and personal health. Therefore, it is essential for destinations to understand the roots of such concerns. SARF, along with its idea of amplification as an attribution, offers a useful framework to understand the process. Thus, for destinations, the baseline is to have a more reflective understanding of the media's role and capability during the crisis and risk communication process. They also need to be familiar with the media content and the existing public perception. The ultimate goal is create effective communication messages that are acceptable to the public.

Additionally, realizing the power of media, CVBs, and officials should not just simply disseminate factual information. The amount of media exposure is an important indicator of the amplification process. Thus, any updates and public campaigns not only need to deliver the most accurate and useful information but also consider visitors' needs and interests. For example, as 
shown in this study, visitors are concerned about the potential impact of Red Tide on their travel experience, which is untestable due to the amount of time, money, and effort they invest in the trip planning process. Therefore, to increase their resistance to crisis events and to maintain their expectations, the messages need to provide realistic solutions such as offering alternative attractions they can enjoy. In fact, Visit Florida employed this strategy by launching a tourism marketing campaign which focused on museums, craft beer, and local experiences, away from the beach focus (DiNatale 2018). Other areas may replicate this strategy by offering alternatives to their closed beaches.

Furthermore, access to communities and the place of residency also influences people's behavioral responses during Red Tide occurrences. Literature in disaster management often points out that upon receiving risk communication, individuals engage in confirmatory actions by contacting their social network to confirm the associated risk and help them in their decisionmaking. This confirmatory action may amplify or attenuate the risk. In addition, because visitors expressed concerns about the risk consequence of their travel experience coupled with the fact that they likely invested significant time and funds for their travel, how these factors mediate visitors' decision-making during conditions of uncertainty warrant further investigation.

For many destinations, domestic tourists are the primary market during the recovery period (Henderson \& Ng, 2004). When it comes to the current context, similarly, Floridians appear to be a valuable market that is essential to Florida's recovery from the negative impacts of Red Tide. More local initiatives should be taken in this regard. Also, due to the importance of access to the community in predicting visitors' behavioral changes, it would be useful to examine how local communities can support visitors, providing them with the necessary resources, re-shaping their risk perception, and educating them about the most accurate self- 
protective behaviors. Built upon the support of local communities, how to effectively utilize residents is another important direction in tourism crisis management, especially since research has found that residents can be very useful during the emergency response phase (Hajibaba, Karlsson, \& Dolnicar, 2017).

\section{Conclusion}

Red Tide and other environmental disturbances are not only challenges for Florida. Recently all Mississippi beaches were closed for several weeks due to toxic algae resulting from a HAB outbreak (Pilie, 2019). Due to the close association between water resources and tourist attractions, the public may generate misperceptions and become hesitant to visit the destination (Liu \& Penington-Grya, 2015). Accordingly, this study helps destinations and practitioners to obtain a better understanding of the process and provides insights into how to manage such situations.

From a theoretical perspective, the findings of this study validated the applicability of SARF to a tourism context and confirmed the importance of the spatial dimension in SARF. Risk perception is a key concept in tourism crisis management research and has been previously linked to specific individual and demographic attributes (Cahyanto \& Pennington-Gray, 2015). The findings of this study advance our understanding in this area, as the study revealed the interaction between media exposure, residency, and the likelihood to engage in protective behaviors. This study also noted visitors' concerns over the perceived consequence of Red Tides on their travel experience, which should be incorporated in the travel risk literature.

This study has a number of limitations. A cross-sectional approach was used with one survey for in-state visitors and another for out-of-state visitors. Although the key variables of the 
samples are similar, the use of two waves of the survey might impede the validity of the findings. On the other hand, it would be interesting to extend these findings using a longitudinal design to assess how media exposure affects individuals' reactions over time. Additionally, this study only measured the extent of media exposure across participants and did not measure the content of the messages portrayed in the media. Further study needs to look at the nature of media messages to fully understand the utility of SARF to understand risk perceptions. Finally, because HAB and Red Tide are commonly occurring worldwide, understanding how different groups such as transient populations perceive such risks and their response warrants further examination to develop an effective risk communication strategy. The influence of culture should also be taken into account. 


\section{References}

Avraham, E., \& Ketter, E. (2008). Media strategies for marketing places in crisis. Journal of Vacation Marketing, 14(4), 373-374.

Brewer, N. T., Chapman, G. B., Gibbons, F. X., Gerrard, M., McCaul, K. D., \& Weinstein, N. D. (2007). Meta-analysis of the relationship between risk perception and health behavior: The example of vaccination. Health Psychology, 26(2), 136-145.

Buttimer, A. (1993). Geography and the human spirit. Johns Hopkins University Press. Cahyanto, I., \& Pennington-Gray, L. (2015). Communicating hurricane evacuation to tourists: Gender, past experience with hurricanes and place of residence. Journal of Travel Research, 54(3), 329-343.

Cahyanto, I., Wiblishauser, M., Pennington-Gray, L., \& Schrouder, A. (2016). The dynamics of travel avoidance: The case of Ebola in the U.S. Tourism Management Perspectives, 20, 195-203.

Chien, P. M., Sharifpour, M., Ritchie, B. W., \& Watson, B. (2017). Travelers' health risk perceptions and protective behavior: A psychological approach. Journal of Travel Research, 56(6), 744-759.

Chong, M., \& Choy, M. (2018). The social amplification of haze-related risks on the Internet. Health Communication, 33(1), 14-21.

Diaz, R. E., Friedman, M. A., Jin, D., Beet, A., Kirkpatrick, B., Reich, A., Kirkpatrick, G., Ullmann, S. G., Fleming, L. E., \& Hoagland, P. (2019). Neurological illnesses associated with Florida Red Tide (Karenia Brevis) blooms. Harmful Algae, 82, 73-81.

DiNatale, S. (2018). What Red Tide? New Florida tourism campaigns promote museums, craft beer and local experience. Tampa Bay Times. https://www.tampabay.com/news/busi ness/tourism/What-Red-Tide-New-Florida-tourismcampaigns-promote-museums-craft-beerand-localexperiences 172501326 
Duckett, D., \& Busby, J. (2013). Risk amplification as social attribution. Risk Management, 15(2), 132-153. https://doi. org/10.1057/rm.2013.2 Florida Division of Emergency Management. (2018). ESF-18 business damage assessment statewide summary report. Southwest Florida Red Tide.

Hair, J. F. (2011). Multivariate data analysis: An overview. In International Encyclopedia of Statistical Science (pp.904- 907). Berlin, Heidelberg: Springer.

Hajibaba, H., Karlsson, L., \& Dolnicar, S. (2017). Residents open their homes to tourists when disaster strikes. Journal of Travel Research, 56(8), 1065-1078. https://doi.org/10.1177/ 0047287516677167

Henderson, J. C., \& Ng, A. (2004). Responding to crisis: Severe acute respiratory syndrome (SARS) and hotels in Singapore. International Journal of Tourism Research, 6(6), 411-419. https://doi.org/10.1002/jtr.505

Hoagland, P., Jin, D., Beet, A., Kirkpatrick, B., Reich, A., Ullmann, S., Fleming, L. E., \& Kirkpatrick, G. (2014). The human health effects of Florida Red Tide (FRT) Blooms: An expanded analysis. Environment International, 68, 144-153. https://doi.org/10.1016/j.envint.2014.03.016

Hornig, S. (1993). Reading risk: Public response to print media accounts of technological risk. Public Understanding of Science, 2(2), 95-109. https://doi.org/10.1088/0963-6625/2/ 2/001

Jonas, A., \& Mansfeld, Y. (2017). Exploring the interplay between the use of risk-related information, risk perception formation, and the stages of travel product consumption. Current Issues in Tourism, 20(14), 1470-1488. https://doi.org/ 10.1080/13683500.2015.1024104 
Karl, M. (2018). The influence of risk perception on destination choice processes. European Journal of Tourism Research, 18, 160-163.

Kasperson, J. X., \& Kasperson, R. E. (Eds.). (2001). Global environmental risk. United Nations University Press. Kasperson, J. X., Kasperson, R. E., Pidgeon, N., \& Slovic, P. (2003). The social amplification of risk: Assessing fifteen years of research and theory. In N. Pidgeon, R.E. Kasperson, \& P. Slovic (Eds)The Social Amplification of Risk, (pp. 13-46). Cambridge: University of Cambridge Press. http://dx.doi. org/10.1017/CBO9780511550461.002

Kasperson, R. E. (2012). The social amplification of risk and low-level radiation. Bulletin of the Atomic Scientists, 68(3), 59-66. https://doi.org/10.1177/0096340212444871 Kasperson, R. E., \& Kasperson, J. X. (1996). The social amplification and attenuation of risk. The Annals of the American Academy of Political and Social Science, 545(1), 95-105.

Kasperson, R. E., Renn, O., Slovic, P., Brown, H. S., Emel, J., Goble, R., Kasperson, J. X., \& Ratick, S. (1988). The social amplification of risk: A conceptual framework. Risk Analysis, 8(2), 178-187. https://doi.org/10.1111/j.1539-6924.1988. tb01168.x

Kozak, M., Crotts, J. C., \& Law, R. (2007). The impact of the perception of risk on international travellers. International Journal of Tourism Research, 9(4), 233-242.

Kuhar, S. E., Nierenberg, K., Kirkpatrick, B., \& Tobin, G. A. (2009). Public perceptions of Florida red Tide Risk. Risk Analysis, 20 (7), 963-969.

Li, Z., Garrison, B., Ullmann, S. G., Kirkpatrick, B., Fleming, L. E., \& Hoagland, P. (2015). Risk in daily newspaper coverage of Red Tide blooms in Southwest Florida. Applied Environmental Education \& Communication, 14(3), 167-177.

Lin, C. A., \& Lagoe, C. (2013). Effects of news media and interpersonal interactions on H1N1 risk perception and vaccination intent. Communication Research Reports, 30(2), 127-136. 
Lindell, M. K., Lu, J. C., \& Prater, C. S. (2005). Household decision making and evacuation in response to Hurricane Lili. Natural Hazards Review, 6(4), 171-179.

Liu, B., \& Pennington-Gray, L. (2015). Bed bugs bite the hospitality industry? A framing analysis of Bed bug news coverage. Tourism Management, 48, 33-42.

Liu-Lastres, B., Schroeder, A., \& Pennington-Gray, L. (2018). Cruise line customers' responses to risk and crisis communication messages: An application of the risk perception attitude framework. Journal of Travel Research, 58(5), 849- 865.

Martin, D. G. (2003). "Place-framing” as place-making: Constituting a neighborhood for organizing and activism. Annals of the Association of American Geographers, 93(3), 730-750.

Masuda, J. R., \& Garvin, T. (2006). Place, culture, and the social amplification of risk. Risk Analysis, 26(2), 437-454.

Matyas, C., Srinivasan, S., Cahyanto, I., Thapa, B., PenningtonGray, L., \& Villegas, J. (2011). Risk perception and evacuation decisions of Florida tourists under hurricane threats: A stated preference analysis. Natural Hazards, 59(2), 871-890.

McCabe, A. S., \& Fitzgerald, M. R. (1991). Media images of environmental biotechnology: What does the public see? In G. S. Sayler, R. Fox, \& A. Blackburn (Eds.), Environmental biotechnology for waste treatment (pp. 15-24).

McKelvey, R. D., \& Zavoina, W. (1975). A statistical model for the analysis of ordinal level dependent variables. The Journal of Mathematical Sociology, 4(1), 103-120. 
Morgan, K. L., Larkin, S. L., \& Adams, C. M. (2011). Empirical analysis of media versus environmental impacts on park attendance. Tourism Management, 32(4), 852-859.

Nierenberg, K., Byrne, M. M., Fleming, L. E., Stephan, W., Reich, A., Backer, L. C., Tanga, E., Dalpra, D. R., \& Kirkpatrick, B. (2010). Florida Red Tide perception: Residents versus tourists. Harmful Algae, 9(6), 600-606.

Park, D. B., \& Yoon, Y. (2009). Segmentation by motivation in rural tourism: A Korean case study. Tourism Management, 30 (1), 99-108.

Pidgeon, N., Kasperson, R. E., \& Slovic, P. (2003). The social amplification of risk. Cambridge University Press. Pilie, S. (2019, July 15). Hurricane Barry didn't wash the algae away from the Mississippi Coast. WGNO ABC News. https:// wgno.com/2019/07/15/hurricane-barry-didntwash-thealgae-away-from-the-mississippi-coast/

Ratzan, S. C., \& Moritsugo, K. P. (2014). Ebola crisis-communication chaos we can avoid. Journal of Health Communication, 19(11), 1213-1215.

Raude, J., Fischler, C., Lukasiewicz, E., Setbon, M., \& Flahault, A. (2004). G.P.s and the social amplification of BSE-related Risk: An empirical study. Health, Risk \& Society, 6(2), 173-185.

Renn, O. (1991). Risk communication and the social amplification of risk. In R.E. Kasperson (Ed.), Communicating Risks to the Public: International Perspectives (pp. 287-324). Dordrecht: Klewer Resnick, B. (2018, October 8). Why Florida's red tide is killing fish, manatees, and turtles. Vox. Retrieved June 26, 2019, from https://www.vox.com/energy-and-environment/2018/ 8/30/17795892/red-tide-2018-florida-gulf-sarasota-sanibelokeechobee

Richter, L. K. (2003). International tourism and its global public health consequences. Journal of Travel Research, 4(4), 340-347. 
Rickard, L. N., McComas, K. A., Clarke, C. E., Stedman, R. C., \& Decker, D. J. (2013). Exploring risk attenuation and crisis communication after a plague death in Grand Canyon. Journal of Risk Research, 16(2), 145-167.

Rubaltelli, E., Scrimin, S., Moscardino, U., Priolo, G., \& Buodo, G. (2018). Media exposure to terrorism and people's risk perception: The role of environmental sensitivity and psychophysiological response to stress. British Journal of Psychology, 109(4), 656-673.

Sarathchandra, D., \& McCright, A. (2017, April-June). The effects of media coverage of scientific retractions on risk perceptions. Sage Open, 7(2), 1-11.

Shakeela, A., \& Becken, S. (2015). Understanding tourism leaders' perceptions of risk from climate change: An assessment of policymaking processes in the Maldives using the Social Amplification of Risk Framework (SARF). Journal of Sustainable Tourism, 23(1), 65-84.

Sims, S. (2018, September 7). A Red Tide on Florida's gulf coast has been a huge hit to tourism. The New York Times. https:// www.nytimes.com/2018/09/07/travel/florida-red-tidetourismgulf-coast.html

Steidinger, K. A. (2009). Historical perspective on Karenia brevis Red Tide research in the Gulf of Mexico. Harmful Algae, 8(4), 549-561.

Thapa, B., Cahyanto, I., Holland, S., \& Absher, J. (2013). Wildfires and tourist behaviors in Florida. Tourism Management, 36, 284-292

Visit Florida. (2018). 2017 Florida visitor study estimates of Florida visitors. https://www.visitflorida.org/resources/ research/visitor-study/ 
Wang, J., Liu-Lastres, B., Ritchie, B. W., \& Pan, D. (2019). Risk reduction and adventure tourism safety: An extension of the risk perception attitude framework (RPAF). Tourism Management, 74, 247-257.

Wilks, J., Stephen, J., \& Moore, F. (Eds). (2013). Managing tourism health and safety in the new millennium. Routledge.

Wirz, C. D., Xenos, M. A., Brossard, D., Scheufele, D., Chung, J. H., \& Massarani, L. (2018).

Rethinking social amplification of risk: Social media and Zika in three languages. Risk Analysis, 38 (12), 2599-2624. https://doi.org/10.1111/risa.13228 